Cinémas

Revue d'études cinématographiques

Journal of Film Studies

\title{
Une reprise impossible? Effi Briest et la question de ses réécritures filmiques
}

\section{Eberhard Gruber}

Volume 4, numéro 1, automne 1993

Écrit/Écran

URI : https://id.erudit.org/iderudit/1000111ar

DOI : https://doi.org/10.7202/1000111ar

Aller au sommaire du numéro

Éditeur(s)

Cinémas

ISSN

1181-6945 (imprimé)

1705-6500 (numérique)

Découvrir la revue

Citer cet article

Gruber, E. (1993). Une reprise impossible? Effi Briest et la question de ses réécritures filmiques. Cinémas, 4(1), 59-71. https://doi.org/10.7202/1000111ar

\section{Résumé de l'article}

Les quatre réécritures filmiques du roman de Fontane, Effi Briest, se trouvent confrontées aux éléments fondateurs des significations fontaniennes que sont : métaphore, temps, reprise. Chaque film les « traite " à sa manière. Ceux de Luderer (1970) et de Jugert (1955) éludent vite la question. Celui de Gründgens (1938) offre l'intérêt de se construire sur une théâtralité que le cinéma s'emploie à exhausser. Seul, le film de Fassbinder (1972) fait porter facture et interrogation filmiques sur la métaphore et le jeu face au réel. Assumant le statut littéraire du récit, le cinéma met dès lors en oeuvre un ensemble de processus spécifiques : fondu au blanc, reprise iconique, jeu de miroirs " glaçant » les personnages, effet de dissociation obtenu par la lecture off du texte ou par son inscription à l'écran. Le film, par suite, toujours déjà réécriture, entre oeuvre et reprise, élabore une polysémie architecturale que fonde tout un art de " rythmer les passages ». 


\title{
Une reprise impossible? Effi Briest $^{1}$ et la question de ses réécritures filmiques
}

\section{Eberhard Gruber}

\author{
pour Anne et Michel Henry
}

\begin{abstract}
RÉSUMÉ
Les quatre réécritures filmiques du roman de Fontane, Effi Briest, se trouvent confrontées aux éléments fondateurs des significations fontaniennes que sont : métaphore, temps, reprise. Chaque film les «traite» à sa manière. Ceux de Luderer (1970) et de Jugert (1955) éludent vite la question. Celui de Gründgens (1938) offre l'intérêt de se construire sur une théâtralité que le cinéma s'emploie à exhausser. Seul, le film de Fassbinder (1972) fait porter facture et interrogation filmiques sur la métaphore et le jeu face au réel. Assumant le statut littéraire du récit, le cinéma met dès lors en œuvre un ensemble de processus spécifiques : fondu au blanc, reprise iconique, jeu de miroirs «glaçant» les personnages, effet de dissociation obtenu par la lecture off du texte ou par son inscription à l'écran. Le film, par suite, toujours déjà réécriture, entre œuvre et reprise, élabore une polysémie architecturale que fonde tout un art de «rythmer les passages».
\end{abstract}

\begin{abstract}
The four film versions of Fontane's Effie Briest confront the basic elements of his signifying systems : metaphor, time and repetition. Each film "handles" these in its own way. The versions by Luderer (1970) and Jugert (1955) quickly evade the question. That by Gründgens (1938) is interesting in being constructed on a theatricality that the cinema strives to rise to. But only Fassbinder's version (1972) bases its technique and exploration on metaphor and play versus the real. Taking on the literary status of a story, the film mobilizes a number of specific
\end{abstract}


procedures : fade to white, iconic repetition, mirrors that "freeze" the characters, a dissociation effect achieved by a voice-off reading of the text or by its inscription on the screen. The film then, already a rewriting, moves between work and repetition, and develops an architectural polysemy grounded in an art of "rhythming the passages."

Me suis crevé les yeux à lire Effi, encore, une page par jour, avec des larmes encore. Effi... aurais pu être heureux avec elle, et les dunes. Non? - Et elle?

Samuel Beckett

La Derniere bande

\section{Quelques éléments pour (re)lire Effi Briest}

C'est une histoire d'adultère que le Dictionnaire des littératures (Larousse) résume comme suit:

Encore adolescente, Effi, mariée au baron Innstetten, de vingt ans son aîné et [haut] fonctionnaire dans un petit port de la Baltique, cède au major Crampas, qui n'est ni beau ni jeune mais sait profiter de la solitude morale de la jeune femme. Sept ans plus tard, à Berlin, un hasard fait découvrir à Innstetten la correspondance des anciens amants; pour satisfaire au code de l'honneur, il tue Crampas en duel et divorce. Rejetée par la bonne société [et mise à la porte par ses parents], Effi retrouvera [trois ans plus tard] la maison de son enfance pour y mourir de la phtisie : en sourdine, tous les harmoniques du réalisme allemand (p. 489).

Voilà, ce que «les gens» veulent entendre et voir illustré "d'habitude», dirait Fontane, tandis que lui développe son roman, justement, «à côté 2 » de ce qui est une «histoire d'adultère comme cent autres ${ }^{3}$ ». Le sujet, rebattu, n'importe que pour et par sa modalisation narrative : "J'écris normalement mieux quand il s'agit d'un non-sujet (Nichtstoff)», dit-il 4 . On l'a compris : «l'accessoire» est "chose principale» pour Fontane 5 , à condition d'entendre là le terme allemand Beiwerk dans lequel le terme «œuvre» (Werk) nous revient «accessoirement». Le «ton» est donné : l'accessoire est à l'«œuvre» ce que le mode est à la chose et le style à la langue.

C'est en ce sens que je propose de faire porter l'accent sur trois aspects du roman : «reprise», «temps», «métaphore».

La liaison entre Effi et Innstetten n'est qu'un remake du rapport de sa mère, Louise, avec le même Innstetten, liaison qui est restée inaboutie à l'époque (I/570sq). «Puisque ce ne pouvait être la mère, il faut que ce soit la fille», dit la vox populi 
(III/577). Ce motif place le roman sous le signe de la "gémellité»: les figures jumelles abondent 6 ; et mis, par Effi, devant la question de savoir qui des deux il préfère d'Effiadolescente ou d'Effi-femme, Innstetten déclare cela «un sujet à thèse», c'est-à-dire insoluble (XXI/724). Gémellité essentielle, donc. D'où vient-elle?

Disons-le d'emblée : du temps même. Le «temps "même"» entendu ici comme un «temps "jumeau"», au sens double du terme : «adjectival», car il n'y a pas de retour temporel sans déplacement; «substantival», car le temps ne peut se manifester que dans la constellation qui forme une figure à deux éléments jumeaux. Le temps-jumeau est, donc, constitutif de la liaison dans laquelle le support fait/est couple. Or, c'est sur ce point précis que se décide le sort des protagonistes. Louise dit à sa fille : en épousant Innstetten, «tu tiendras, à vingt ans, un rang auquel d'autres accèdent à quarante» (II/575). Il s'agit, donc, de «gagner du temps sur le temps» (de gagner contre le temps) et de refuser ainsi que le temps soit un partenaire. C'est cette ambition, qui est vouée à l'échec, selon Fontane, tout comme l'ascension sociale où s'aliènent amour et bonheur. Tout le monde est conscient de cette distorsion, admet que Louise aurait été «mieux assortie avec Innstetten» (V/593; IX/624), et Effi au cousin Dagobert (V/594sq; XXI/724). Mais les deux femmes nient la distorsion (V/595 et XXI/724; III/576). C'est pourquoi il ne faut pas employer trop vite le terme de «tragique» qui sousestime l'apport actif de chacun/e.

Mais comment décide-t-on du «temps»? Quand Effi consent à l'union avec Innstetten, elle s'engage à une sorte d'uniformité : «Tout homme convient», dit Effi, au début, "pourvu qu'il soit noble, dispose d'une situation et ait belle allure» (III/577). Or, le propre des femmes, dira un personnage dans L'Adultera (1882), est qu'elles «ne supportent pas l'uniformité (Einerlei) ni dans le quotidien ni dans le bonheur ${ }^{7}$ ». Effi n'est donc «pas un être féminin» quand elle accepte le mariage 8 et elle ne le devient qu'au prix d'événements dits tragiques. «Respecter le temps», par contre, c'est faire avec le temps jumeau; «doubler le règne du monotone» et prévenir, ainsi, l'infortune.

Autrement dit : au temps jumeau qui ne se perçoit qu'à travers une gémellité réelle, on ne peut répondre que par un équivalent ou par l'oubli; par la présentation de la gémellité ou par sa distorsion. C'est dire qu'on entre sur le terrain de la représentation du temps. Où il faut distinguer entre «uniformité dominante» (qu'est l'oubli du temps jumeau) et «temps jumeau retrouvé» grâce à un couplage réel. Mais, surtout, où il appert 
que toute compréhension passe par les métaphores du temps (c'est-à-dire les constructions qui l'exhibent).

Qu'est-ce qui se dit à travers la métaphore? Effi-adolescente et Effi-femme sont-elles, face au temps, plutôt de l'ordre de la «métaphore», portant sur un comparant ou phore «unique», à l'image de l'intégralité de l'individu, ou sont-elles, plutôt, de l'ordre de l'«allégorie» qui porte, à l'image des stades différents d'une personne, sur "plus d'un" phore (cf. GRADUS)? Je soulève le problème, sans le résoudre; car ce n'est pas la métaphore en elle-même qui m'intéresse, ici, mais «ce» qu'elle «veut dire». Le «corps» de la «métaphore» n'importe qu'en ce qu'il est le support d'un mouvement qui se «dégage» de lui, «le dépasse» : c'est ce que méta-phore signifie, «porter d'(un lieu) à (un autre)» ou trans-port(er).

Soit. À cette précision près toutefois : la métaphore, face au temps jumeau qui conduit à des formations jumelles, ne «quitte» un lieu que pour y «revenir». Le temps ne l'accueille que pour la renvoyer à sa place qui n'est ni vraiment la même ni vraiment la sienne. La métaphore, par suite, doit compter avec sa «pareille» : pareille à «elle-même» dans la comparaison d'un instant à l'autre; pareille à «son autre» dans la soustraction du métaphorique en faveur du sens propre.

Et le temps pendant ce temps? «Le» temps a dis-paru, «paru» à travers le dis-/le duel des constructions qui le manifestent. «Parution/disparition» qui, par itérativité, intermittence et sens propre de la métaphore, implique un fonctionnement qui porte à une transposition. Le «fonctionnement», en effet, relie les extrêmes que sont "présence», «absence», «interruption», «retour» et constitue ainsi un processus de «transposition» : tels les mouvements contradictoires d'un balancier ou d'une balançoire.

Par quoi nous retournons, d'un trait, au cœur d'Effi Briest. Car, tout l'art de Fontane consiste à tresser son roman selon une orientation qui joue, en terme de métaphore, l'oubli du temps jumeau contre sa reconnaissance : le sort d'Effi est, d'entrée, étroitement associé à la «balançoire», lieu qui symbolise son plus grand bonheur et, plus tard, une mélancolie éclairée d'espoir. Et durant toute sa vie conjugale, elle est animée d'une inquiétude qui n'a de fin qu'avec sa mort : «Le calme», dit-elle, «le calme», peu avant de mourir (XXXVI/828). Or, "calme» en allemand se dit Ruhe tandis qu'«inquiétude» c'est Unruhe dont le deuxième sens signifie «balancier» 9 .

Examinons le choix de la métaphore : la «balançoire» produit jeu et plaisir, fait un va-et-vient tout en restant au lieu même des 
mouvements contradictoires. Ce fonctionnement est analogue à celui de la métaphore, car celle-ci est à sa présence, à son absence et à son retour ce que la balançoire est aux positions différentes que son mouvement parcourt. La balançoire, par ailleurs, ne nécessite aucun partenaire : seul entre en jeu l'élan de celui qui se balance - ce qui donne une certaine souveraineté à l'individu face aux autres et, surtout, face au temps. Effi fait corps avec son élan et répond ainsi à l'injonction du temps"même». Fontane le dit à sa manière : Effi, "mettant en mouvement la planchette de la balançoire par le jeu habile de son corps qui se dressait et se baissait rapidement et en souplesse» (XXXIV/816; nous soulignons), constitue une sorte de "ressort interne» qui fait fonctionner la balançoire. Elle est donc son propre «balancier». Privilégier la valeur métaphorique de la balançoire, c'est aller au plus subtil du rapport de l'individu au temps.

Pré-figuration donc, que cette métaphore. Car dès la première page, l'ensemble s'esquisse magistralement : bien que la demeure des Briest soit inondée de soleil, l'ombre portée du bâtiment ne rejoint encore ni le massif comprenant, en son milieu, un cadran solaire ni, plus loin, la balançoire. Toutefois, les sombres événements sont déjà inscrits : afin de se balancer, Effi doit se placer entre deux cordes qui tiennent la planchette dont «l'une» fait penser à la corde du pendu à propos de quoi Crampas, futur amant d'Effi, lance une boutade prémonitoire ${ }^{10}$, tandis que «l'autre» corde est associée à la cordelette rouge qui entourera la fatale liasse de lettres, preuve de l'adultère (XXVI/768). Le sort, littéralement suspendu ici, annonce son issue juste à côté : le cadran solaire fera place au tombeau d'Effi dont la plaque de marbre blanc portera son nom du début, de jeune fille (sans celui du baron Innstetten); scène d'une disparition (ou ré-apparition?) que figure déjà «la petite porte de fer peinte en blanc», unique interruption (et passage obligé) entre le jardin des Briest et le mur du cimetière voisin (I/565; manque dans la traduction Villain $)^{11}$. Et partout, déjà, se répand le lierre, plante mortuaire qui, par son nom allemand, Efeu, rampe «littéralement» après Effi. C'est à cause des voyelles «e et $i$ », dit Fontane, qu'il a choisi le nom d'Effi Briest; mais c'est, surtout, par le jeu présence/absence d'une lettre que Fontane produit, ici, du sens ${ }^{12}$. Tout se joue à travers la différence entre Efeu et Effi; d'autant plus que le même procédé pèse, par la présence ou absence d'une consonne cette fois, celle du $r$, sur le nom de famille : Briest et Biest opposent, en effet, le nom du père à celui de la «garce» ou «bête»13. 
Cette façon de composition narrative soulève la question de savoir si le style fontanien contrebalance vraiment les avantsignes mortuaires. Face au soleil et à l'ombre, qui des deux porte plus loin : le tombeau ou la balançoire? Certes, Effi meurt de la phtisie, mais au moins autant de l'imitation sociale qui n'est, au fond, que celle de l'oubli du temps. N'est-elle pas gratifiée d'un «talent d'imitation» (XXXIV/813)? Elle en meurt. Effi, victime de mimèsis, donc. Mais de quoi vit-elle, tout de même?

\section{Tant que la métaphore (trans)porte}

La métaphore «mime» en un surplace le temps-jumeau. Elle n'est que $r e$-présentation du temps. D'où la question : quel élément «dépasse» la métaphore «balançoire» tout en lui étant constitutif? C'est celui qu'Effi, en se balançant, ressent de façon agréable : l'«air» (II/573; XXXIV/816 et passim). «Sylphide», «fille de l'air» dit d'elle sa mère (I/566). Mais que penser de cette ascendance si Effi phtisique en meurt?

Dans un grand monologue avant que n'éclate le scandale, Effi s'interroge : comment peut-elle "avoir une faute sur (l') âme», sans que cela lui "pèse» (XXIV/758)? La «faute» et sa «remise» dépendent donc de ce que le "vouloir-dire» de la métaphore «fait le poids» ou "non». C'est à cette condition, seulement, qu'on peut parler, malgré la «faute», d' «innocentement», la «faute» étant comme trans-posée, contrebalancée par.

Voici la scène : après une sorte de prière intérieure, Effi «baissa» la tête, pleura, et «lorsqu'elle se redressa (...), redevenue plus calme (...), des platanes arrivait à son oreille un son, doux et fin, comme s'il pleuvait»(XXIV/759). Fontane figure ici une intervention qui, «venant entre» les mouvements contradictoires d'Effi, agit en littérale «méta»-physique ${ }^{14}$, permettant de se demander si ce qui s'annonce par un «son», n'a pas la valeur d'un bénéfique partenaire pour Effi. À quoi le narrateur donne réponse sept lignes plus loin : «(...) sur les platanes s'épanchait, après comme auparavant, un bruissement semblable à celui d'une pluie fine», avant de conclure : "Mais ce n'était que l'air de la nuit qui marchait» (die Nachtluft, die ging : XXIV/759). Autrement dit : face à tous les soucis d'Effi, l'air fait le poids parce qu'il représente la mobilité même qui (em)porte. Pour obtenir cet effet, Fontane mêle bruissement de l'air à pesanteur de la pluie et lie ces deux volets par un «comme», signe de la qualité porteuse. L'air, qui a aussi du poids et de la mobilité comme, signale l'interférence concrète de ce qu'il symbolise. Cette lecture prend tout son sens à la fin du roman : «(...) plus [Effi] tendait l'oreille, plus nettement elle entendait, à nouveau, que ça (es) tombait (niederfiel), comme 
une pluie fine, sur les platanes.» Mais, au lieu d'expliquer, comme précédemment que ce n'était qu'une brise nocturne, le narrateur, par une «ellipse», donne tout le «poids» nécessaire «à l'air» et ne rapporte que «l'effet» produit : «Un sentiment libérateur s'empara d'elle. "Le calme, le calme", dit-elle» (XXXVI/828). Derniers mots d'Effi - qui est, est-ce hasard, un mot double. Ou jumeau.

De quel ordre est cet élément, dont parle l'«air»? Il ne relève pas de l'humain, ni d'une séparation, ni d'une domination. Si c'était le cas, Effi qui a entendu autrefois le «son infiniment subtil», de la mer, "presque comme une voix humaine» (XIX/703), aurait su le reconnaître. Le «son de l'air» qu'elle vient d'entendre n'est rapportable ni à l'eau ni à la voix : ce qui fait (ré)sonner l'air c'est un lien non-humain et libérateur qui, selon la logique de toute liaison, «reporte» en un autre lieu 15 . Qui ou quoi trans-porte? Le lien est à ce qu'il lie ce que le mode est à ce qu'il modèle : Effi, en mourant, se donne à son mode d'être; c'est-à-dire ni à elle-même (au sens d'un état réifié), ni à un autre (fût-il «Dieu»), mais à ce que la métaphore de l'air «figure»: le trans-port qui fait partie de la vie «même»; «l'élément transfigurateur» qui conduit au retour par un déplacement et fait que Effi ne disparaît... qu'en apparence. Quand Fontane parle de la «transfiguration»(Verklärung), il s'intéresse plutôt au réancrage dans le réel qu'au sens religieux de ce terme ${ }^{16}$. Le réel transfiguré c'est «le va-et-vient non aliéné (unverzerrtes Widerspiel) de la vie que nous menons»17. Autrement dit : Effi part, ayant été; et elle sera, parce que partie.

Quel que soit le jugement qu'on porte sur cette fin (ou sur sa lecture), il faut retenir que Fontane joue à fond le jeu de la polysémie avec la métaphore; car, attribuer à l'air mouvement et pesanteur, c'est dire que la balançoire ne fonctionne, en sa juste mesure, que par l'apport de l'air. Fontane signale ainsi l'intervention de ce qui nous arrive, tel un don, de l'extérieur, et nous est bénéfique. L'air représente ce don d'un "partenaire»: et c'est l'ouverture.

\section{Défaire, refaire une polysémie ouverte : les réécritures filmiques d'Effi Briest}

Les quatre réécritures filmiques d'Effi Briest ont un point commun : «l'omission» des passages du texte qui signalent l'élément transfigurateur et sa manifestation métaphorique. Dès lors, la pluralité non saturable des significations fontaniennes se réduit à la subjectivité d'un auteur-interprète.

Écartons d'abord rapidement deux films de peu d'intérêt : Effi Briest de Wolfgang Luderer, tourné en 1970 pour la télévision 
en RDA, se construit sur une téléologie du social qui enlève à la langue, à l'individu et à sa volonté tout poids historique. (La suppression des derniers mots de Crampas, tué en duel, "Voulezvous...», est emblématique à cet égard (XXVIII/780). Il reste du roman une carcasse du passé, reconstruite avec un pédantisme pédagogique qui propose une Effi (Angelica Domröse) moitié scout moitié vamp et la punit de ses charmes par une chute de balançoire (ajoutée au texte). Plus grossière encore est la version de Rudolf Jugert qui, sous le titre incongru de Rosen im Herbst («Des roses en automne», 1955/RFA) ne cherche que l'exploitation commerciale et transforme le roman en une histoire où Effi (Ruth Leuwerik) meurt, coupable de n'avoir pas donné à Innstetten l'amour qui lui manquait. Épitaphe inventée qui ouvre et clôt le film : "L'amour est la forme suprême de la religion» (!).

Mieux vaut examiner les deux films qui restent proches du roman. Gustaf Gründgens, qui produit, en 1938, la première adaptation pour l'écran, prend pour titre de son film la pièce de Ernst Wichert (1872) et que Crampas, dans le roman, met en scène (XVIII/691sq) : Der Schritt vom Wege, «Un pas hors du chemin», c'est-à-dire «Un écart» ou encore «Un faux pas»18. Titre qui donne au récit un aspect de «comédie»: on ne verra que théâtre. Le film s'ouvre et s'achève sur le même plan : les parents d'Effi assis sur un banc, face à la caméra, à bonne distance d'observation, semblent regarder, tel un vrai public, les scènes, à suivre. Ayant choisi de dramatiser, Gründgens s'emploie à opposer la vitalité naturelle d'Effi (Marianne Hoppe) à la conformité sociale d'Innstetten. Considérons deux scènes, inventées, indicatrices du style de Gründgens. La première se situe au début : Effi est en train de se balancer; Innstetten, réserviste en exercice, passe en uniforme blanc, avec un régiment de hussards; ils se saluent par-dessus le mur qui sépare le jardin de la rue. Innstetten, dans ce costume, est comparé à Lohengrin par la boutade d'Effi. La seconde scène démonte, plus tard, la première : un jour, Effi prie son mari de se remettre en uniforme blanc pour lui faire plaisir; lorsqu'il apparaît en «faux Lohengrin", Crampas, vrai major, bientôt son rival, vient de faire son entrée. Théâtralité appuyée d'Innstetten : faux soldat et faux acteur, il est aussi faux mari et fausse autorité. En fait, Gründgens, le plus souvent, n'utilise le théâtral que pour exhausser ses effets. Tout le côté douloureux propre à Effi Briest est oblitéré.

Au contraire, c'est sur la métaphore et le jeu face au réel que porte l'interrogation de Fassbinder. Le metteur en scène assume 
le statut «littéraire» de l'histoire dont il conserve le texte fidèlement. S'il refuse la transfiguration - ce qu'il nomme "atmosphère sécurisante» (Geborgenheit) ${ }^{19}$ —, il a le mérite de créer une œuvre équivalente à celle de Fontane et de mettre le doigt sur le problème de la «reprise» - je vais y revenir.

«Pour moi, c'est une tentative pour mettre en scène la littérature (Literaturverfilmung). C'est la littérature qui est le vrai sujet du film.». L'important ne sera donc pas l'histoire mais «le comment raconter 20 ». Il en résulte, de la part de Fassbinder, une sorte de mise à plat de la narration filmique. Les mots du roman sont repris par la voix d'un narrateur (la sienne), par les dialogues des protagonistes et par quatorze intertitres qui sont des citations et qui exhaussent le textuel. Fassbinder produit un film à structure parataxique où les scènes se succèdent sans développement : point démontré par le cours du montage, le «drame» est admis comme antérieur. Dès la première image, tous les liens sont cassés. Et Effi (Hanna Schygulla), sur sa balançoire, bouge à peine. Scènes très lentes, statiques, véritables tableaux vivants - ou plutôt morts. L'absence de mouvement se conjugue à l'absence d'émotion. Quant aux protagonistes, pas davantage de liens : tout mouvement vers l'autre se fige en pose; pas de rapport à son propre corps que dédouble le miroir; distance à la langue qu'acteurs et actrices récitent plus qu'ils ne la parlent. En somme : il n'y a pas de jeu — à tous les sens du terme. Seuls les nombreux fondus au blanc «entrecoupant» les scènes font événement : "Le fondu au blanc», dit Fassbinder, a pour fonction de «réveiller l'intellect» du spectateur; il situe le film dans sa facture, "comme si on lisait un livre et tournait la page ou commençait un chapitre nouveau 21 ». Le «blanc» c'est le «vide» qui déborde toute scène, mais sans offrir d'alternative. Fassbinder ajoute au titre du film un long sous-titre, Fontane Effi Briest ou À propos de tous ceux qui pressentent leurs possibilités et leurs besoins et qui, malgré tout, acceptent dans leur tête et par ce qu'ils font, le système dominant et en conséquence, le renforcent et le confirment parfaitement sous-titre où il s'implique lui-même : «Fontane porte sur le monde une vue où je me reconnais, et qu'on peut bien sûr condamner, à savoir que les choses sont comme elles sont et qu'on ne peut les changer que très difficilement. Bien qu'on sache qu'il faut les changer, un jour, on en perd l'envie (Lust), et on se borne, tout simplement, à les décrire 22 ». Cette façon de marquer l'impasse où se dit, désarmé et désarmant, le désir d'un dépassement, semble être propre à notre époque (on se rappelle 
du refus de Fassbinder de cautionner le groupe Baader/Meinhof); Fontane indique au contraire un mode d'être : qui «laisse être».

Que reste-t-il de cette mise en scène de la littérature? Il reste l'art de la construction fontanienne, défaite car non transfigurée. Il reste la tenue de la langue même si dépouillée du vouloir-dire métaphorique. Il reste le triste vis-à-vis des éléments narratifs, des espaces, des personnages. Froide atmosphère d'uni-vers parallèles que renforce le motif d'une gémellité tenant les uns et les autres à distance, et chacun de soi-même. À cet effet, Fassbinder utilise surtout, avec une insistance impressionnante, les jeux de miroirs qui divisent le plan, "glacent» et figent les personnages. Tantôt on ne voit que reflets. Tantôt on se rend compte après coup que l'image montrait un reflet "parlant» ou un tableau-portrait dissocié de toute voix 23 . D'où l'importance de la langue qui reste, malgré tout, le seul lien tangible. Langue qui continue à (nous) parler lorsque, à l'écran, le tombeau d'Effi ne porte ni nom ni croix (à la différence de ce que dit le texte : XXXVI/828sq) : la plaque de marbre blanc est représentée par une «image blanche» (l'écran lui-même) sur laquelle s'inscrit la ligne noire d'un cadre rectangulaire. Ce «cadre vide» indique, certes, qu'un personnage fictif ne meurt pas de la même manière qu'un personnage réel. Le cadre vide souligne aussi que la mort en littérature c'est le retrait vers les constituants élémentaires : le «blanc» (du support), le «noir» (de l'inscription), pré-cédant tout récit - qui en sont la trame aussitôt oubliée. Le message du film est aussi vide que l'écran est blanc : tout est image déclarée. Le seul espoir de changement ne peut venir que de l'ici : tout est à faire. La mort du personnage fictif «Effi» n'est qu'un indice à l'adresse du spectateur, pour qu'il (y) réfléchisse. Résultat de l'honnêteté intellectuelle du metteur en scène qui expose le problème sans anticiper quelque solution. Ceci est à l'opposé de la polysémie cathartique de Fontane.

En somme, on peut dire que les quatre versions filmiques d'Effi Briest rabattent, chacune différemment, la polysémie ouverte du roman sur un sens propre : "théâtral» pour Gründgens, «commercial» pour Jugert, «historico-idéologique» pour Luderer et critico-sceptique pour Fassbinder.

Il conviendra, pour terminer, de resserrer le questionnement sur le rapport œuvre-reprise qui est au cœur du problème écrit/écran.

\section{Du sens de la réécriture/écriture : œuvre et reprise}

C'est aux trois sens de «reprise» que doit s'entendre la réécriture : «reprendre» quelque chose; la «raccommoder»; 
participer à la polysémie de l'œuvre tel un «refrain», au sens musical.

Si l'œuvre, voire le chef-d'œuvre, est question de jeu polysémique ouvert, ne sera à la hauteur qu'une réécriture capable de donner le change en réactivant l'ouverture sur la pluralité de l'œuvre.

Autrement dit : entre l'œuvre qui lui préexiste et sa propre ouverture, la reprise comporte un déplacement; elle ne saurait opérer sur le même lieu. Dès lors, la position des versions filmiques se dessine mieux. Posons, d'abord, que toute réécriture consiste à jeter une sorte d' «écran» sur l'œuvre afin d'y inscrire «sa» version. Entrent en jeu les deux sens d'«écran» : 1) support de projection; 2) élément intermédiaire. Or, ce qui est capital dans la situation-cinéma c'est le recul face au support-images recul qui protège le support comme tel et permet la projection. Recul et support, l'écran fait ainsi fonction d'ouverture : ouverture sur les images par rapport à quoi il constitue à la fois une «distance inaliénable» et la «matérialisation» de cette distance qui est un retrait. De ce fait, en somme, on peut dire que l'écran est à l'image ce que l'ouverture est à la polysémie d'un roman comme Effi Briest.

Gründgens et Fassbinder, je l'ai dit, opèrent de façon opposée, chacun faisant porter l'accent sur une partie (une moitié) du roman : l'un transforme le livre en métaphore théâtrale; l'autre souligne le manque de jeu. Pour Gründgens, qui profite à fond de l'ouverture filmique sans jamais la questionner, les événements en restent à l'image ou à la figure de théâtre; tandis que Fassbinder qui projette «rien» — c'est-à-dire la cause même de l'angoisse —, inscrit, sur l'image qu'il défait et refait jusqu'en sa trame, des effets dévastateurs et l'injonction que «ça change». Ce faisant, Fassbinder objecte à Fontane - qui trouve appui sur l'arrière-fond du récit et sur la fonction donative de la narration en retrait —, que rien n'est à reprendre, au fond, car ce qui pourrait valoir la peine (si tant est que quelque chose de cet ordre puisse être envisagé) reste encore à faire.

Écoutons, une fois encore, l'Effi Briest de Fontane :

Les battants inférieurs des fenêtres étaient restés ouverts, et les petits rideaux blancs se gonflaient dans le courant d'air ( $\mathrm{Zug}$, der ging) et retombaient doucement par-dessus le dossier de la chaise, jusqu'à ce que vienne un autre courant d'air (ein neuer Zugwind $\mathrm{kam})$ qui les libérait à nouveau (XXIV/757).

S'expose, ici, à la manière de la balançoire, l'itérativité des extrêmes alternés : venir-aller (kam und ging) au bord d'une ouverture («fenêtre») dans un espace délimité et non. Itérativité 
selon un rythme qui interrompt chaque mouvement pour renvoyer à l'autre, et ainsi de suite. C'est à ce «rythme» que tient le roman Effi Briest, rythme qui dépasse tout mouvement, toute motion et émotion. Ce que Gründgens a compris en rythmant son film par de longues et belles prises sur les dunes, le ciel, la mer. Cadence du temps. Pour le reste, tout ce qui n'est pas rythme est mise en scène.

En deçà de l'eschatologie qui se dessine ici, il est temps de récapituler, c'est-à-dire de rappeler ceci : qu'à la puissance de transfiguration de l'élément métaphorique, chez Fontane, générateur de polysémie et réplique au rythme, répond le pouvoir de l'opération filmique par quoi l'écran-support transfigure l'écran-distance, l'image, l'écran-support, le récit, l'image. Comme si l'œuvre livresque portait avec/en soi l'attente d'une confrontation de ses images, mots, sons - confrontation fondée sur la reprise, le refrain, et porteuse d'autres images, mots, sons. On peut supposer que l'œuvre filmique capable de reprendre la problématique de l'écriture fontanienne serait celle qui, conjuguant le scepticisme de Fassbinder et la théâtralité de Gründgens pour qu'ils forment couple, s'emploierait à rythmer leur réciproque interruption. Cette injonction à se dépasser, à se trans-figurer revient par suite à «rythmer les passages»: entre aller et venir, entrer et sortir, fictif et réel. En conséquence, «l'atmosphère sécurisante» propre à Fontane ne serait ni à imposer ni à réfuter mais à reprendre. Ce que Fassbinder, à sa manière, fait au niveau élémentaire par les fondus au blanc (où la projection offre un nouveau rebond à l'image), mais ne fait pas au niveau thématique où la tâche de vitaliser la vie revient au spectateur critique.

Toute écriture, qu'elle soit textuelle ou filmique, est, toujours déjà, sous le coup de l'ouverture, la reprend et la berce, l'expose, la joue d'un bout à l'autre, la raccommode, et n'est que pré-texte à sa continuation, par itérativité et reprise. Autrement dit : de même que la «réécriture» a lieu entre sa propre ouverture et l'œuvre précédente, de même «l'écriture» a lieu entre le rythme de sa venue et ses retours. Écriture «e(s)t» reprise : où «e(s)t» s'entend à la fois comme auxiliaire et conjonction - «e(s)t» qui «rythme». C'est bien de cela qu'il s'agit quand Effi, à la fin, se donne au coup de vent (Zugwind) qui porte calme et paix. Zugwind est aussi ce «courant qui em-porte» : Effi «est» parce qu'Effi part d'un coup de «et». C'est de cela dont parle Fontane : de l'air — porteur de rythme. 


\section{NOTES}

1 Nous citons le roman de Theodor Fontane (1895) dans la traduction de Pierre Villain aux Éditions Robert Laffont, 1981, pp. 561-829, en indiquant d'abord le chapitre et, après la barre, la page. La traduction est très souvent remaniée. Pour l'accès aux quatre versions filmiques, je remercie Mme Brigitte Capitain du Deutsches Institut für Filmkunde (DIF) à Francfort/Main.

2 Voir ce que dit le personnage Ebba dans le roman Unwiederbringlich (1892 : «Irréparable» ou, mieux, «Irréversible»), chapitre XXII.

3 Lettre à Friedrich Spielhagen du 21 février 1896.

4 Lettre à Emilie Fontane du 17 août 1882. C'est Fontane qui souligne.

5 Lettre à Theodor Wolf du 24 mai 1890.

6 On trouve dans le roman de «vraies» jumelles (Hertha et Bertha : I/567), des jumelles littéraires (Mining et Lining : ib.), de simples ressemblances (Louise et Effi : XXIII/741; Louise et Annie, sa petite fille : $\mathrm{XXX} / 762$ ) et des jumeaux par âge et jour d'anniversaire (Louise et Innstetten : $\mathrm{I} / 569)$.

7 Theodor Fontane, L'Adultera (Paris : Aubier, 1991) chap. XVI, p. 140 (nous remanions la traduction de Madith Vuaridel).

8 Le «oui» d'Effi n'est, d'ailleurs, pas rapporté par le texte (II/575).

9 L'étude de Peter Demetz «Vol de flocon» («Flug und Flocke», in : id., Formen des Realismus. Theodor Fontane, München : 1964, pp. 204-216), développant l'aspect thématique, est complémentaire de notre approche. Je remercie Lucien Dällenbach de m'avoir indiqué ce texte.

10 «Qui mérite la corde, ne peut périr à l'eau» dit Crampas (XV/672), par quoi le roman fait penser à cet autre dicton «Qui mérite le duel, ne peut mourir au gibet».

11 Cf. la traduction d'André Cœuroy republiée aux Presses d'aujourd'hui : 1981, p. 1.

12 Lettre à Julius Rodenberg du 9 novembre 1893. Quant à l'absence/présence de «e» et «i» voir aussi le jeu de mot du cousin d'Effi (widerfahren/wieder fahren : «advenir»/«conduire à nouveau»; XXIII/737).

13 Voir aussi la valeur «lugubre» que le narrateur attribue à la voyelle $u$ (I/572), en affirmant ainsi l'«art de l'antithèse» (V/591).

14 Méta- signifie, en grec, aussi «entre».

15 Voir à cet égard les passages où la question du «son» se pose sous les aspects indiqués (XIX/703; XX/711; IX/624).

16 Voir la transfiguratio du Christ sur le mont Thabor (Math. XVII).

17 Remarque publiée posthume par Joseph Ettlinger (éd.) Aus dem Nachlass von Theodor Fontane (Berlin : 2/1908) p. 236. C'est Fontane qui souligne.

18 Fontane publie une critique de cette pièce de Wichert dans la Vossische Zeitung du 1 nov. 1872.

19 Rainer Werner Fassbinder, Die Anarchie der Phantasie (Frankfurt/M. : Éd. S. Fisher, 1986) p. 56 (entretien avec Kraft Wetzel du juillet 1974).

20 Entretien avec Corinna Brocher in Der Tagesspiegel du 11 février 1972 et (sous un autre titre) in Stuttgarter Zeitung du 1 décembre 1972.

21 Rainer Werner Fassbinder, Die Anarchie der Phantasie, p. 55.

22 Entretien Brocher/Fassbinder.

23 Par exemple dans le premier dialogue entre Effi et Johanna, l'employée, à Kessin; ou dans l'entretien d'Innstetten et Wüllersdorf qui est décisif pour le duel. Signalons en outre que Fassbinder fait doubler la voix de plusieurs acteurs. 